\title{
Inflammatory expression profiling defines the tumor microenvironment and immunity in lower-grade gliomas
}

\section{Hua Yang}

Department of Neurosurgery, The Affiliated Hospital of Guizhou Medical University

\section{Xin Xiang}

Department of Neurosurgery, The Affiliated Hospital of Guizhou Medical University

\section{Yaka Xu}

Department of Neurosurgery, The Affiliated Hospital of Guizhou Medical University

\section{Ze-Jing Zhao}

Clinical medicine, Guizhou Medical University

\section{Han Peng}

Department of Neurosurgery, The Affiliated Hospital of Guizhou Medical University

\section{Yi-Min Chen}

Department of Neurosurgery, The Affiliated Hospital of Guizhou Medical University

\section{Wen-Yan Li}

Department of Neurosurgery, The Affiliated Hospital of Guizhou Medical University

\section{Jie Yang}

Department of Neurosurgery, The Affiliated Hospital of Guizhou Medical University

\section{Xiao-Lan Qi}

Key Laboratory of Endemic and Ethnic Diseases, Ministry of Education \& Key Laboratory of Medical Molecular Biology of Guizhou Province

\section{Chun-De You}

Department of Neurosurgery, The Affiliated Hospital of Guizhou Medical University

\section{Liang-Zhao Chu}

Department of Neurosurgery, The Affiliated Hospital of Guizhou Medical University

\section{Xing-Wang Zhou ( $\square$ aliya7@126.com )}

Department of Neurosurgery, The Affiliated Hospital of Guizhou Medical University

\section{Research Article}

Keywords: Lower-grade glioma, Inflammatory response, Immune infiltration, Tumor microenvironment

Posted Date: February 22nd, 2022 
DOI: https://doi.org/10.21203/rs.3.rs-1362715/v1

License: (c) (1) This work is licensed under a Creative Commons Attribution 4.0 International License. Read Full License 


\section{Abstract}

Objective: Lower-grade glioma (LGG) arises from glial or precursor cells including the world health organization (WHO) grade glioma, which has a favorable prognosis compared with glioblastomas. However, LGG progression is inevitable due to drug resistance and tumor invasiveness. The inflammatory response has been a well-established hallmark associated with tumor occurrence and recurrence. It is necessary to explore the heterogeneity of the inflammatory response in LGG.

Methods: The differential and prognostic inflammatory response-related genes(IRRG) of the cancer genome atlas(TCGA) database were used to perform consensus clustering to identify robust clusters. The Chinese Glioma Genome Atlas(CGGA) database was used to validate this inflammation subtype. The clinical characteristics, immune infiltration, metabolism, and somatic variation of each cluster were assessed. A risk score associated with inflammation was constructed.

Results: We successfully observed and validated three distinct inflammatory subtypes in LGG. It is noteworthy that the $B$ subtype has a shorter overall survival than the $A$ and $C$ subtypes. The $B$ subtype had higher immune, stromal, and ESTIMATE scores than the $A$ and $C$ subtypes but lower tumor purity than the $A$ and $C$ subtypes. In addition, immune, stromal, and ESTIMATE scores of the A subtype were higher than the hose of the $\mathrm{C}$ subtype, while tumor purity was lower than that of the $\mathrm{C}$ subtype. Three inflammation subtypes correlated with diverse metabolic characteristics, drug sensitivity, and somatic alterations. Furthermore, we developed and validated an inflammatory signature with better performance of prognosis prediction.

Conclusion: This study identified a new classification of LGG according to inflammatory gene profiling and emphasized inflammatory heterogeneity within LGG.

\section{Introduction}

Lower-grade glioma(LGG) arises from glial or precursor cells and includes world health organization(WHO) grade gliomas, such as astrocytoma, oligodendrogliomas, anaplastic astrocytomas, anaplastic oligodendrogliomas, and anaplastic oligoastrocytomas ${ }^{1,2}$. LGG was classified into IDH (isocitrate dehydrogenase) mutant, IDH mutant, and 1p19q deleted, and IDH wildtype gliomas according to the IDH mutation and 1p19q status ${ }^{3,4}$. According to the pathological type and tumor grade of LGG, the current therapeutic schedule includes surgical resection, radiotherapy, and chemotherapy. However, tumor progression is inevitable due to drug resistance and tumor invasiveness ${ }^{5-7}$.

The inflammatory response is a well-established hallmark of cancer ${ }^{8,9}$. It is estimated that $15-20 \%$ of all deaths from cancer worldwide are associated with the inflammatory response ${ }^{10}$. Inflammatory cells and inflammatory mediators are hallmarks of cancer-associated inflammation. A biological effect of cancerrelated inflammation is the one that follows cancer progression ${ }^{10}$. Most malignancies induce an inflammatory response that builds up a protumorigenic microenvironment to promote cancer cell- 
autonomous proliferation, invasion, angiogenic switch, and metastases ${ }^{10-12}$. Inflammation may produce cytokines and chemokines to recruit leukocytes and lymphocytes ${ }^{13}$, induce oncogene mutation, inhibit cell apoptosis, and finally lead to the activation of the inflammatory signal transduction pathway. Furthermore, most immunosuppressive cells, including M2-TAMs, MDSCs, and Tregs, are recruited by tumor-related inflammation, which may provide more convenience for the construction of the immunosuppressive tumor microenvironment and regulate malignant tumor biological behavior ${ }^{14-16}$. The value of peripheral blood inflammatory markers, including the neutrophil-to-lymphocyte ratio, monocyte-to-lymphocyte ratio ${ }^{17}$, systemic immune-inflammation index ${ }^{18}$, prognostic nutritional index, and postoperative controlling nutritional status ${ }^{19}$, have been evaluated in glioma patients, and those inflammatory markers were associated with the prognosis of glioma patients. The inflammatory microenvironment may indisputably play an important role in the proliferation and progression of glioma. Inflammation-related genes have been used to predict prognosis and the tumor microenvironment ${ }^{20}$. However, the clinical relevance, tumor microenvironment, somatic mutation, and drug sensitivity among distinct LGG subtypes based on the inflammatory expression profile should be revealed, which may lead to improved diagnosis and prognosis of LGG.

In the present study, LGG was classified into three distinct tumor types according to the inflammatory response expression profiles via unsupervised clusters. Each subtype had different clinical relevance, tumor microenvironment, metabolism, somatic mutation, and drug sensitivity. Furthermore, we constructed and validated a risk score based on the differentially expressed genes among the three subtypes of LGG with better performance of prognosis prediction.

\section{Methods}

\section{Data extraction and preparation}

We extracted RNA-seq data, somatic mutations, and the corresponding clinical information of LGG from The Cancer Genome Atlas (TCGA) dataset (http://cancergenome.nih.gov/). Two RNAseq datasets and one microarray dataset were downloaded from the Chinese Glioma Genome Atlas (CGGA, http://www.cgga.org.cn) while obtaining the corresponding clinical information. The two RNAseq datasets were merged and named CGGA1, and the microarray dataset was named CGGA2. The transcriptional group gene expression of normal brain tissues was extracted from the GTEX dataset by using the UCSC website ${ }^{43}$ (http://xena.ucsc.edu/). Furthermore, 200 inflammatory response-related genes were identified from the GSEA database (http://www.gsea-msigdb.org/gsea/index.jsp).

\section{Identification of differential and prognostic inflammatory response-related genes (IRRG)}

The differentially expressed inflammatory genes were obtained by using the "Bioconductor Limma" $\mathrm{R}$ package to assess the expression of glioma and normal tissues. Genes with a false discovery rate $<0.05$ and a fold change $>2$ were defined as differentially expressed genes ${ }^{44}$. After that, the "survival" 
$R$ package was employed to evaluate the association between differential genes and the overall survival of LGG patients. A gene with $\mathrm{p}<0.05$ was considered a prognosis-related gene.

\section{Construction and validation of inflammatory response subtypes (IRS)}

The differential IRRGs were selected to perform consensus clustering through the R package "consensusClusterplus" 45 . The cumulative distribution function and consensus heatmap were used to identify the optimal k. The TCGA database was used as a training cohort, while CGGA1 and CGGA2 were used as validation cohorts. The R package "clusterRepro" was used to assess the similarity and reproducibility of the IRS between the training cohort and validation cohort.

\section{Tumor Immune Microenvironment}

To qualify the infiltration level of 23 immune cells, we used the R package "GSVA" to conduct a single-sample gene-set enrichment analysis (ssGSEA) ${ }^{46}$. Furthermore, ESTIMATE was used to estimate the immune score, stromal score, and tumor purity 47 .

\section{Computation of metabolism-associated gene signatures}

A total of 115 metabolism gene signatures were extracted from previously published studies ${ }^{48}$. Then, the 115 scores of the metabolism-associated signatures for each sample were estimated by performing the R package "GSVA".

\section{Construction and validation of the inflammation-related signature}

First, we identified the genes with differential expression among IRSs in TCGGA, CGGA1, and CGGA2 by using the R package "samr". The R package "Veen" was employed to identify the intersection genes among those three cohorts ${ }^{49}$. Second, the intersection genes with differential expression and prognostic relevance were identified as candidate genes to perform the Cox proportional hazards model using the R package "glmnet". Finally, we used the regression coefficients to estimate the risk score of each sample. The TCGA cohort was the training cohort, and CGGA1 and CGGA2 were validation cohorts $^{50}$.

\section{Drug sensitivity analysis}

We used the R package "pRRophetic" to calculate the drug sensitivity for each IRS. The Dependent Cancer Drug Sensitivity Genomics (GDSC) database was employed to achieve the maximum inhibitory concentration (IC50) and prediction accuracy via 10-fold cross-validation and ridge regression ${ }^{51}$.

\section{Statistical analysis}

All statistical analyses were performed using R software (version 4.0.4). A chi-square test was carried out to compare the clinical and molecular features between subtypes. One-way ANOVA was 
performed for the comparison of three groups. The survival among IRS was evaluated by Kaplan-Meier analysis with a log-rank test. Univariate and multivariate analyses were conducted to obtain the factors associated with the prognosis of LGG patients. Statistical significance was identified as $p$ value $<$ or $=$ 0.05 .

\section{Results}

\section{Identification of prognosis-related differential inflammatory genes}

A total of 200 inflammatory genes were identified from the GSEA database. Ninety-eight genes were differentially expressed between 356 normal tissues and 529 tumors. Forty-eight genes were upregulated, while 50 were downregulated. Seventy of those 98 genes were associated with the prognosis of LGG patients, as revealed by survival analysis. Then, the 70 prognosis-related differential inflammation genes were employed to perform a consensus cluster (Figure 1a and b).

\section{A consensus cluster was performed to divide glioma into distinct subtypes.}

To assess the heterogeneity of inflammation in LGG, we conducted a consensus cluster and identified three IRSs (A, B, and C) based on 70 prognosis-related differential inflammatory genes (Figure $1 \mathrm{c}$ and $\mathrm{d}$ ). The TCGA database was used as a training cohort, while CGGA1 and CGGA2 were used as testing cohorts. We used PCA to evaluate the robust difference in expression characteristics within these three inflammatory subtypes (Figure 2). It is noteworthy that the B subtype had a shorter overall survival than the $\mathrm{A}$ and $\mathrm{C}$ subtypes in the training cohort (Figure 2).

We used CGGA1 and CGGA2 to validate the results obtained in the TCGA database and evaluate the reproducibility and stability in the testing cohort by calculating the centroid of each IRS. Pearson correlation of centroid was employed to assign the samples to a subtype in CGGA datasets. The results showed that the IRSs of TCGA and CGGA were highly consistent (Table 1). The same prognostic information for IRS was obtained from the CGGA1 and CGGA2 cohorts (Figure S1 and S2).

\section{Correlation of the IRSs with clinical characteristics}

To evaluate the clinical relevance of IRS, the relationship with molecular and pathological features was determined. We found that anaplastic astrocytoma, IDH wild-type, 1p19q noncodeletion, and WHO III grade were associated with the B subtype, while WHO II grade, IDH mutation, and 1p19q codeletion were associated with the $A$ and $B$ subtypes (Figure $2 a$ ). We also obtained similar clinical correlations from the CGGA database(Figures S1 and S2, Tables S3, S4).

\section{Immune infiltration of IRSs in LGG}

Due to the close relationship between inflammation and immunity, we calculated the immune infiltration of each IRS by SSGSEA. We observed that all 23 immune cells were highly enriched in the $B$ subtype (Figure $3 a$ and $3 c$ ). The A subtype had a median enrichment of 23 immune cells, and the $C$ 
subtype had low enrichment. Moreover, ESTIMATE was used to estimate the immune score, stromal score, and tumor purity. The results showed that the B subtype had higher immune, stromal, and ESTIMATE scores than the $A$ and $C$ subtypes but lower tumor purity than the $A$ and $C$ subtypes. In addition, the immune, stromal, and ESTIMATE scores of the A subtype were higher than those of the $C$ subtype, while tumor purity was lower than that of the $C$ subtype (Figure $3 b$ ). All of these results suggested that the $B$ subtype was an immunogenic tumor, and $C$ was a nonimmunogenic tumor. We also validated the above results in the CGGA1 and CGGA2 cohorts, and similar results were obtained (Figure S3 and S4). In addition, we also assessed the distribution of CTLA4, PDCD1, and HLA gene expression among IRSs (Figure 4). We found that the B subtype had higher CTLA4, PDCD1, and HLA gene expression than the $A$ and $C$ subtypes, respectively, and the gene expression of the $A$ subtype was higher than that of the $C$ subtype (Figure 5).

\section{Metabolic characteristics of IRSs}

Metabolic changes were associated with inflammation and tumor development ${ }^{21}$. A total of 115 metabolism gene signatures of each sample were calculated to explore the metabolic characteristics of each IRS. Differential analyses indicated that higher levels of metabolism processes in the B subtype were mainly carbohydrate, vitamin, and nitrogen metabolism compared with the $A$ and $C$ subtypes. However, the $C$ subtype displayed a high level of metabolism processes that were associated with lipid, nucleotide, and oxidative phosphorylation, whereas ADP-ribosylation and carbohydrate metabolism were enriched in the A subtype when the two subtypes were compared with each other (Figure 6). We further computed those metabolic scores in the CGGA1 and CGGA2 cohorts and identified similar results (Figure S5 and S6).

\section{IRSs were associated with diverse somatic variations}

The inflammatory response within the tumor microenvironment may be regulated by genetic aberrations. As a result, we further explored the association between IRSs and diverse somatic variations. A subtype was enriched in DIH1, TP53, CIC, and ATRX mutations. The B subtype was enriched in mutations in driver genes such as IDH1, TP53, TTN, ATRX, EGFR, and PTEN. However, DIH1, TP53, and ATRX mutations were highly enriched in the $C$ subtype. Furthermore, we calculated the tumor burden for each sample. We observed that the B subtype had a higher tumor burden than the $A$ and $C$ subtypes. (Figure 7).

\section{The potential therapeutic value of IRSs}

To identify the potential therapeutic value of IRSs, we explored the relationship between IRSs and drug sensitivity. The results indicated that AMG.706, docetaxel, and KIN001.135 showed a high sensitivity for the A subtype. Twenty-eight drugs showed drug sensitivity associated with B subtypes, such as CEP.701, EHT.1804, and elesclomol. However, 56 drugs exhibited varying degrees of sensitivity associated with $\mathrm{C}$ subtypes, such as dasatinib, erlotinib, and cyclopamine. Together, these results showed that the inflammatory response was associated with drug sensitivity (Figure 8 and Table S5). 


\section{Construction of an inflammatory signature with prognostic significance}

We next constructed a prognostic signature with a Cox proportional hazards model according to the inflammatory expression classification we developed. First, we identified 3383, 4035, and 562 differentially expressed genes among IRSs(A vs. B, B vs. C, A vs C) from TCGA, CGGA1, and CGGA2 cohorts, respectively (Figure 9a, b, and C). Then, a total of 209 intersection genes were obtained from TCGA, CGGA1, and CGGA2 cohorts, and differential analyses and Cox proportional hazards models were used to select potential genes (Figure 9d, e, and f). Subsequently, we identified 13 genes (TNFRSF12A, TGIF1, IL15RA, ADAP2, GRIN3A, APOBEC3G, PLAUR, RNF149, ITPRIP, HLA. DOA, NCKAP1 L, CD14, FGL2) with differential expression and prognostic value to construct the inflammatory signature. Kaplan-Meier analysis showed that LGG patients with low scores had longer overall survival (Figure 10a and b). Multivariate Cox regression analysis also indicated that the inflammatory signature was an independent prognostic factor for LGG patients (Figure S7). In addition, the risk score had a high AUC in predicting the overall survival of LGG patients (Figure 10c and f, Figure S8). We further validated this inflammatory signature in the CGGA dataset, and consistent results were obtained (Figure 10d, e, and f, Figure S8).

\section{Discussion}

The inflammatory response is a well-established hallmark of cancer ${ }^{8,9}$. Recently, an accumulating body of research indicated that the tumor microenvironment is characterized by a persistent inflammatory response, which is regarded as the "wound that does not heal" (Inflammation and cancer: back to Virchow?). Inflammatory cells, inflammatory cytokines, and inflammation-related signaling pathways within the inflammatory microenvironment may provide a good material preparation for tumor progression and then lead to poor prognosis of $\mathrm{LGG}^{22-24}$. Therefore, it is of great clinical significance to explore the heterogeneity of the inflammatory response in the LGG microenvironment, which may provide insights into inflammatory tumor microenvironment targeting. In the present study, we established three IRSs through unsupervised clustering based on inflammatory expression profiling and transcriptomics data. The clinical features, immune infiltration, metabolic characteristics, somatic variations, and drug response of each IRS were assessed. Our study may deepen our understanding of inflammatory heterogeneity and extend the molecular subtyping of LGG.

Increasing evidence suggests that inflammation may have an impact on proliferation, metastases, immune surveillance and treatment response ${ }^{25}$. Systemic inflammatory markers, including the neutrophillymphocyte ratio (NLR), platelet-lymphocyte ratio (PLR), and monocyte lymphocyte ratio (MLR) are associated with the clinical outcome of many kinds of malignant tumors ${ }^{26-31}$. Recently, the effect of inflammation-related genes in malignant tumors was fully evaluated by using public databases, such as TCGA and GEO ${ }^{20,32}$. The inflammation signature constructed according to the inflammatory genes was associated with clinical outcomes and immune infiltration of several tumors ${ }^{20,32}$. In this study, we classified LGG into three distinct subtypes based on prognosis-related differential inflammatory genes. We found that inflammation-related genes in the B subtype were high expression, $C$ subtype was low 
expression while A subtype was median expression. Survival analysis indicated that the B subtype showed a shorter overall survival than the $A$ and $C$ subtypes, which implied that an active inflammatory response may contribute to tumor progression.

Inflammation has many connections with immunity 8,9 . Although both immunity and inflammation constitute the basic characteristics of the tumor microenvironment, the relationship between them is still very vague. It is speculated that in the early stage of tumor development, the immune response is strong, and inflammation is weak, but in the later stage of the tumor, the immune response is weak, while inflammation is strong. However, because the tumor microenvironment is extremely complex and in a changing process, it is necessary to further study the cells, molecules, and internal mechanical signals $\mathrm{s}^{33}$. Due to the different inflammatory forms, the tumor microenvironment not only includes cancer cells and their surrounding matrix (fibroblasts, pericytes, mesenchymal cells, endothelial cells) but also innate immune cells (including dendritic cells, mast cells, natural killer cells, macrophages, neutrophils, and myeloid suppressor cells) and adaptive immune cells ( $T$ and $B$ lymphocytes) ${ }^{34}$. These cells are interconnected through direct contact or the production of cytokines and chemokines and then control and shape tumor growth in autocrine and paracrine ways ${ }^{35}$. In this study, we identified that the B subtype was characterized by a high level of immune cell infiltration, which suggested that the B subtype was a hot immune tumor. A subtype also had a higher immune cell infiltration than the $C$ subtype. The immune, stromal, and ESTIMATE scores of the A subtype were higher than those of the $\mathrm{C}$ subtype, while tumor purity was lower than that of the $C$ subtype. Although antitumorigenic immune cells, such as natural killer cells, $B$ cells, and CD8 + T cells, were increased in the $C$ subtype, the balance based on the expression of various immune mediators and modulators as well as the abundance and activation state of different cell types in the tumor microenvironment is profoundly tilted towards protumor inflammation ${ }^{9,36}$. How to reverse this balance by regulating the transformation of the tumor microenvironment to promote the effect of immunotherapy may be a worthy topic for further studies.

Inflammation is closely related to metabolic diseases ${ }^{37-39}$, while the relationship between metabolism and inflammation in the tumor environment remains unknown. Regarding the metabolic characteristics of IRSs, we observed that higher levels of metabolism processes in the B subtype were mainly carbohydrate, vitamin, and nitrogen metabolism compared with the $A$ and $C$ subtypes. However, the $C$ subtype displayed a high level of metabolism processes that were associated with lipid, nucleotide, and oxidative phosphorylation, whereas ADP-ribosylation and carbohydrate metabolism were enriched in the A subtype when the two subtypes were compared. Previous studies have shown that metabolism is associated with clinical outcomes and has potential prognostic value $e^{40-42}$. Metabolic processes, including carbohydrate, vitamin, and nitrogen metabolism, which were upregulated in the B subtype, were associated with poor prognosis, while lipid metabolism, which was upregulated in the $C$ subtype, was reported to be related to favorable clinical outcomes in LGG and other cancers ${ }^{40}$. Further studies were performed to explore the relationship between the inflammatory response and metabolism of the tumor environment in vitro and in vivo. 
IDH and 1p19q status have been used in the molecular subtype classification of gliomas ${ }^{4}$. When we associated IRSs with IDH mutation and 1p19q in the TCGA cohort, 7.58\% (27/356) of IDH mutations and $4.05 \%(6 / 148)$ of $1 p 19 q$ codeletion tumors were enriched in B subtypes. In all IDH mutation tumors, $51.69 \%$ (184/356) were assigned to the A subtype, while $40.73 \%(145 / 356)$ were assigned to the $C$ subtype. Furthermore, $25 \%$ (37/148) of all $1 \mathrm{p} 19 \mathrm{q}$ codeleted tumors were enriched in the A subtype, whereas $70.95 \%$ (105/148) were classified into the $C$ subtype. In addition, the intersection genes among IRSs were used to construct the IRscore, and a high IRscore was associated with poor clinical outcomes. CGGA datasets were used to validate these results, and similar results were obtained. Our study may broaden our understanding of the molecular subtyping of LGG.

In conclusion, we classified LGG from inflammatory expression profiling and proposed three subtypes with distinct clinical outcomes: immune infiltration, metabolism, and somatic variation. Our results demonstrated the inflammatory heterogeneity of LGG and may provide a valuable classification for the development of inflammation-targeted therapies. However, further studies need to performed to explore the underlying mechanism between inflammation and immune infiltration and metabolism.

\section{Declarations}

\section{Acknowledgment}

None.

\section{Author information}

These authors contributed equally: Hua Yang, Xin Xiang, Yaka Xu.

Affiliations

Department of Neurosurgery, The Affiliated Hospital of Guizhou Medical University, Guiyang, Guizhou province, P.R.China

Hua Yang, Xin Xiang, Yaka Xu, Yi-Min Chen, Wen-Yan Li, Jie Yang, Chun-De You, Liang-Zhao Chu, XingWang Zhou and Han Peng

Clinical Medicine, Guizhou Medical University, Guiyang 550004, Guizhou, China

Ze-Jing Zhao

Key Laboratory of Endemic and Ethnic Diseases, Ministry of Education \& Key Laboratory of Medical Molecular Biology of Guizhou Province, Guizhou Medical University, Guiyang 550004, Guizhou, China

Xiao-Lan Qi

Contributions 
X.-W.Z., L.-Z.C., H.Y., and X.X. were responsible for the original draft writing, acquisition, investigation, conceptualization, visualization, and software. X.-W.Z., K.X., and M.-H.D. were involved in the methodology. X.-W.Z., Y.-M.C., W.Y.L., J.Y., X.-L.Q., Z.-J.Z., H.P. and C.D.Y. were involved in the conception and design of the study and revised the manuscript. All authors have read and agreed to the final version of the manuscript.

Corresponding authors

Correspondence to Liang-Zhao Chu(365446506@qq.com) or Xing-Wang Zhou(aliya7@126.com)凹Department of Neurosurgery, The affiliated Hospital of Guizhou Medical University, Guiyang, Guizhou province, P.R.

\section{Funding}

No.

\section{Data Availability Statement}

The datasets presented in this study can be found in online repositories. Further inquiries can be directed to the corresponding authors.

\section{Ethics declarations}

Competing interests

The authors declare no competing interests.

Ethics approval

All data were retrieved from TCGA and CGGA datasets; therefore, no informed consent was necessary.

\section{References}

1. Louis, D. N. et al. The 2007 WHO classification of tumours of the central nervous system. Acta neuropathologica 114, 97-109 (2007).

2. Ostrom, Q. T. et al. CBTRUS Statistical Report: Primary Brain and Other Central Nervous System Tumors Diagnosed in the United States in 2012-2016. Neuro-Oncology 21, V1-V100 (2019).

3. DJ, B. et al. Comprehensive, Integrative Genomic Analysis of Diffuse Lower-Grade Gliomas. The New England journal of medicine 372, 2481-2498 (2015).

4. Louis, D. N. et al. The 2016 World Health Organization Classification of Tumors of the Central Nervous System: a summary. Acta neuropathologica 131, 803-820 (2016).

5. Huang, L. E. Friend or foe-IDH1 mutations in glioma 10 years on. Carcinogenesis 40, 1299-1307 (2019). 
6. Appolloni, l. et al. Progression from low- to high-grade in a glioblastoma model reveals the pivotal role of immunoediting. Cancer letters 442, 213-221 (2019).

7. Youssef, G. \& Miller, J. J. Lower Grade Gliomas. Current neurology and neuroscience reports 20, (2020).

8. Mantovani, A., Allavena, P., Sica, A. \& Balkwill, F. Cancer-related inflammation. Nature 454, 436-444 (2008).

9. Grivennikov, S. I., Greten, F. R. \& Karin, M. Immunity, inflammation, and cancer. Cell 140, 883-899 (2010).

10. Balkwill, F. \& Mantovani, A. Inflammation and cancer: back to Virchow? Lancet (London, England) 357, 539-545 (2001).

11. Soucek, L. et al. Mast cells are required for angiogenesis and macroscopic expansion of Mycinduced pancreatic islet tumors. Nature medicine 13, 1211-1218 (2007).

12. Swann, J. B. et al. Demonstration of inflammation-induced cancer and cancer immunoediting during primary tumorigenesis. Proceedings of the National Academy of Sciences of the United States of America 105, 652-656 (2008).

13. Sparmann, A. \& Bar-Sagi, D. Ras-induced interleukin-8 expression plays a critical role in tumor growth and angiogenesis. Cancer cell 6, 447-458 (2004).

14. Whiteside, T. L. FOXP3 + Treg as a therapeutic target for promoting anti-tumor immunity. Expert opinion on therapeutic targets 22, 353-363 (2018).

15. Zhang, J. et al. Tumoral NOX4 recruits M2 tumor-associated macrophages via ROS/PI3K signalingdependent various cytokine production to promote NSCLC growth. Redox biology 22, (2019).

16. Weber, R. et al. IL-6 as a major regulator of MDSC activity and possible target for cancer immunotherapy. Cellular immunology 359, (2021).

17. Chim, S. T., Sanfilippo, P., O’Brien, T. J., Drummond, K. J. \& Monif, M. Pretreatment neutrophil-tolymphocyte/monocyte-to-lymphocyte ratio as prognostic biomarkers in glioma patients. Journal of neuroimmunology 361, 577754 (2021).

18. Luo, Y. et al. The prognostic value of inflammation markers in postoperative gliomas with or without adjuvant treatments. Medicine 100, e26437 (2021).

19. Yılmaz, H., Niğdelioğlu, B., Oktay, E. \& Meydan, N. Clinical significance of postoperatif controlling nutritional status (CONUT) score in glioblastoma multiforme. Journal of clinical neuroscience: official journal of the Neurosurgical Society of Australasia 86, 260-266 (2021).

20. Han, T. et al. Comprehensive Analysis of Inflammatory Response-Related Genes, and Prognosis and Immune Infiltration in Patients With Low-Grade Glioma. Frontiers in Pharmacology 12, 1-15 (2021).

21. Chou, W. C., Rampanelli, E., Li, X. \& Ting, J. P. Y. Impact of intracellular innate immune receptors on immunometabolism. Cellular and Molecular Immunology (2021).

22. Müller, S. et al. Single-cell profiling of human gliomas reveals macrophage ontogeny as a basis for regional differences in macrophage activation in the tumor microenvironment. Genome biology 18 , 
(2017).

23. Greten, F. R. \& Grivennikov, S. I. Inflammation and Cancer: Triggers, Mechanisms, and Consequences. Immunity 51, 27-41 (2019).

24. Albrengues, J. et al. Neutrophil extracellular traps produced during inflammation awaken dormant cancer cells in mice. Science (New York, N.Y.) 361, (2018).

25. Greten, F. R. \& Grivennikov, S. I. Inflammation and Cancer: Triggers, Mechanisms, and Consequences. Immunity 51, 27-41 (2019).

26. Wang, Y. et al. The prognostic significance of preoperative neutrophil-lymphocyte ratio in patients with hepatocellular carcinoma receiving hepatectomy: A systematic review and meta-analysis. International journal of surgery (London, England) 55, 73-80 (2018).

27. Zhu, Y., Zhou, S., Liu, Y., Zhai, L. \& Sun, X. Prognostic value of systemic inflammatory markers in ovarian Cancer: a PRISMA-compliant meta-analysis and systematic review. BMC cancer $18,(2018)$.

28. Mori, K. et al. Prognostic value of preoperative blood-based biomarkers in upper tract urothelial carcinoma treated with nephroureterectomy: A systematic review and meta-analysis. Urologic oncology 38, 315-333 (2020).

29. Wiencke, J. K. et al. Immunomethylomic approach to explore the blood neutrophil lymphocyte ratio (NLR) in glioma survival. Clinical epigenetics 9, (2017).

30. Graziano, V. et al. Combination of peripheral neutrophil-to-lymphocyte ratio and platelet-tolymphocyte ratio is predictive of pathological complete response after neoadjuvant chemotherapy in breast cancer patients. Breast (Edinburgh, Scotland) 44, 33-38 (2019).

31. Kawai, M. et al. Low lymphocyte monocyte ratio after neoadjuvant therapy predicts poor survival after pancreatectomy in patients with borderline resectable pancreatic cancer. Surgery $165,1151-$ 1160 (2019).

32. Xie, Z. et al. Development and Validation of Prognostic Model in Transitional Bladder Cancer Based on Inflammatory Response-Associated Genes. Frontiers in Oncology 11, 1-12 (2021).

33. Hui, L. \& Chen, Y. Tumor microenvironment: Sanctuary of the devil. Cancer letters 368, 7-13 (2015).

34. De Visser, K. E., Eichten, A. \& Coussens, L. M. Paradoxical roles of the immune system during cancer development. Nature reviews. Cancer 6, 24-37 (2006).

35. Lin, W. W. \& Karin, M. A cytokine-mediated link between innate immunity, inflammation, and cancer. The Journal of clinical investigation 117, 1175-1183 (2007).

36. Smyth, M. J., Dunn, G. P. \& Schreiber, R. D. Cancer immunosurveillance and immunoediting: the roles of immunity in suppressing tumor development and shaping tumor immunogenicity. Advances in immunology 90, 1-50 (2006).

37. Saltiel, A. R. \& Olefsky, J. M. Inflammatory linking obesity and metabolic disease and metabolic disease. Journal of Clinical Investigation 127, 1-4 (2017).

38. Hotamisligil, G. S. N F L Amm a T I O N a N D M E T a B O L I C D I S O R D E R S. Insight Review Nature (2006). 
39. Zamani-garmsiri, F. Polyphenols: Potential anti-inflammatory agents for treatment of metabolic disorders. 1-18 (2021).

40. Wu, F. et al. Metabolic expression profiling stratifies diffuse lower-grade glioma into three distinct tumour subtypes. British Journal of Cancer 125, 255-264 (2021).

41. Kim, J. \& DeBerardinis, R. J. Mechanisms and Implications of Metabolic Heterogeneity in Cancer. Cell metabolism 30, 434-446 (2019).

42. Peng, X. et al. Molecular Characterization and Clinical Relevance of Metabolic Expression Subtypes in Human Cancers. Cell reports 23, 255-269.e4 (2018).

43. Lonsdale, J. et al. The Genotype-Tissue Expression (GTEx) project. Nature genetics 45, 580-585 (2013).

44. Wu, F. et al. Molecular classification of IDH-mutant glioblastomas based on gene expression profiles. Carcinogenesis 40, 853-860 (2019).

45. Yu, G., Wang, L. G., Han, Y. \& He, Q. Y. clusterProfiler: an R package for comparing biological themes among gene clusters. Omics: a journal of integrative biology 16, 284-287 (2012).

46. Hänzelmann, S., Castelo, R. \& Guinney, J. GSVA: gene set variation analysis for microarray and RNASeq data. BMC Bioinformatics 14, 7 (2013).

47. Yoshihara, K. et al. Inferring tumour purity and stromal and immune cell admixture from expression data. Nature communications 4, (2013).

48. Rosario, S. R. et al. Pan-cancer analysis of transcriptional metabolic dysregulation using The Cancer Genome Atlas. Nature communications 9, (2018).

49. Chen, H. et al. Cross-talk of four types of RNA modification writers defines tumor microenvironment and pharmacogenomic landscape in colorectal cancer. Molecular Cancer 20, (2021).

50. Remark, R. et al. Characteristics and clinical impacts of the immune environments in colorectal and renal cell carcinoma lung metastases: Influence of tumor origin. Clinical Cancer Research 19, 40794091 (2013).

51. Geeleher, P., Cox, N. \& Stephanie Huang, R. pRRophetic: an R package for prediction of clinical chemotherapeutic response from tumor gene expression levels. PloS one 9, (2014).

\section{Figures}

\section{Figure 1}

The expression of inflammation-related genes and consensus clustering based on inflammation associated with gene expression in the TCGGA dataset. a: The heatmap showed that 98 inflammationassociated genes were differentially expressed between 356 normal tissues and 529 tumors; b: Volcano 
plots showed that forty-eight genes were upregulated while 50 were downregulated; c: clustering matrix for $\mathrm{k}=3$; $\mathrm{d}$ : delta area.

\section{Figure 2}

Identification of distinct IRSs in LGG through inflammatory gene profiling. a: Heatmaps of three IRSs defined in TCGA cohorts and the relationship between IRSs and clinical features. b: Principal component analysis of three IRSs using candidate genes. c: Survival analyses show significant differences between the three IRSs in TCGA cohorts.

\section{Figure 3}

Immune infiltration and tumor microenvironment of IRSs in the TCGA cohort. a: Heatmap of IRSs associated with immune infiltration and immune function; b: tumor microenvironment of IRSs; $c$ : the signature of 23 immune cells among IRSs.

\section{Figure 4}

HLA gene expression among IRSs. a: TCGA; b: CGGA1; c: CGGA2.

\section{Figure 5}

CTLA4 and PDCD1 expression among IRSs. a: TCGA; b: CGGA1; c: CGGA2.

\section{Figure 6}

The metabolic characteristics of IRSs in the TCGA database. a: A vs. B; b: A vs. C; c: B vs. C.

\section{Figure 7}

Distinct somatic mutations in IRSs. a: A subtype; b: B subtype; c: C subtype; $d$ : tumor mutation burden of IRSs. 


\section{Figure 8}

Drug sensitivity associated with IRSs. a, b, and c show that dasatinib, erlotinib, and cyclopaine were sensitive to the $C$ subtype; $d$, e and $f$ indicate that CEP.701, EHT.1864, and elesclomol were sensitive to the B subtype.

\section{Figure 9}

The intersecting genes among IRSs from TCGA, CGGA1, and CGGA2 cohorts. a, b, c: The common genes of IRSs observed from TCGA, CGGA1, CGGA2 cohorts, respectively; d: the common genes of IRSs observed from TCGA, CGGA1, CGGA2 cohorts; e: the heatmap of different expression levels of intersection genes between glioma and normal tissues; f: volcanic map showing the up- and downregulated common genes.

\section{Figure 10}

The development and validation of the IR score in TCGA and CGGA1 cohorts. a, d: The 14 genes expressed between the high and low IR score groups in TCGA and CGGA1, respectively; b, e: The prognostic role of the IR score in TCGA and CGGA1, respectively; $c$, $f$ : The predictive role of the IR score in TCGA and CGGA1, respectively.

\section{Supplementary Files}

This is a list of supplementary files associated with this preprint. Click to download.

- SupplementalFiles.docx 